\title{
Chiral Corrections to Nucleon Electromagnetic Form Factors Calculated in the NJL Model
}

\author{
Robert J. Perry* \\ CSSM and ARC Centre of Excellence for Particle Physics at the Tera-scale, Department of \\ Physics, University of Adelaide, Adelaide SA 5005, Australia \\ E-mail: robert.perryeadelaide.edu.au

\section{Anthony W. Thomas} \\ CSSM and ARC Centre of Excellence for Particle Physics at the Tera-scale, Department of \\ Physics, University of Adelaide, Adelaide SA 5005, Australia \\ E-mail: anthony.thomaseadelaide.edu.au
}

\section{Manuel E. Carrillo-Serrano}

CSSM and ARC Centre of Excellence for Particle Physics at the Tera-scale, Department of Physics, University of Adelaide, Adelaide SA 5005, Australia

E-mail: manuel.carrilloserrano@student.adelaide.edu.au

\begin{abstract}
It has long been known that corrections motivated by chiral symmetry arguments give important contributions to hadronic observables. Results of incorporating pion loop corrections to the NJL Model - a constituent quark model - are presented here. A particular parameter set yields a good fit to the nucleon charge radius $\left\langle r_{N}^{2}\right\rangle$ and a slightly low prediction of the nucleon magnetic moment $\mu$.
\end{abstract}

The 26th International Nuclear Physics Conference

11-16 September, 2016

Adelaide, Australia

\footnotetext{
* Speaker.
} 


\section{Introduction}

The elastic electromagnetic form factors of the nucleon are observables of chief interest since they may be related to the electric and magnetic charge distributions of the particle, and they therefore give information about the structure of the nucleon in question. As a result, improved measurements of the nucleon electromagnetic form factors have been the subject of ongoing experimental programs. While much good data exists for the proton, the lack of a free target makes the determination of neutron's electromagnetic form factors more difficult, and in this case, one is forced to consider other methods for determining electromagnetic form factors.

In the present work, the NJL Model formalism developed previously [1, 2, 3] is modified to recalculate nucleon electromagnetic form factors, where pion cloud effects are incorporated using the Light Front Cloudy Bag Model. The results of this approach are compared to the previous method, to elucidate differences between the two. The outline of this procedings is as follows. Section 2 discusses briefly the NJL Model and the calculation of electromagnetic form factors, Section 3 describes the alternative method, employed in this procedings, with the results of this approach presented in Section 4, and finally the conclusion is given in Section 5.

\section{Nucleon Form Factors in the NJL Model}

The Nambu-Jona-Lasinio (NJL) model is a well known constituent quark model. The Lagrangian density for the $S U(3)$ flavour NJL Model, in its Fierz symmetric form is given as [3]

$$
\mathscr{L}=\bar{\psi}(i \not \partial-\hat{m}) \psi+\frac{1}{2} G_{\pi}\left[\left(\bar{\psi} \lambda_{i} \psi\right)^{2}-\left(\bar{\psi} \gamma_{5} \lambda_{i} \psi\right)^{2}\right]-\frac{1}{2} G_{\rho}\left[\left(\bar{\psi} \gamma^{\mu} \lambda_{i} \psi\right)^{2}+\left(\bar{\psi} \gamma^{\mu} \gamma_{5} \lambda_{i} \psi\right)^{2}\right]
$$

where $\hat{m}=\operatorname{diag}\left(m_{u}, m_{d}, m_{s}\right)$ and $\lambda_{i}$ are the eight generators of $S U(3)$ in the Gell-Mann Representation, plus $\lambda_{0}=\sqrt{2 / 3}$.

Previously, baryon electromagnetic form factors have been calculated in the NJL Model [2, 3], where baryons are naturally described as quark-diquark bound states [2]. The electromagnetic form factors are defined by the electromagnetic current $j^{\mu}$ :

$$
\left\langle p^{\prime}, s^{\prime}\left|j^{\mu}\right| p, s\right\rangle=\bar{u}\left(p^{\prime}, s^{\prime}\right)\left[\gamma^{\mu} F_{1}\left(Q^{2}\right)+\frac{i \sigma^{\mu v} q_{v}}{2 m_{N}} F_{2}\left(Q^{2}\right)\right] u(p, s),
$$

where $p, s$ are the momentum and spin states of the nucleon. Note that, as is conventional in the literature, $Q^{2}=-q^{2}$. In the implementation of the NJL Model used here, the quark-photon vertex is dressed by including contributions from vector meson dominance, and importantly for this procedings, pion loop effects.

It is common to use the Sachs Parameterization of the electromagnetic form factors, which are given as linear combinations of $F_{1}$ and $F_{2}$;

$$
\begin{aligned}
& G_{E}\left(Q^{2}\right)=F_{1}\left(Q^{2}\right)-\frac{Q^{2}}{4 m_{N}^{2}} F_{2}\left(Q^{2}\right), \\
& G_{M}\left(Q^{2}\right)=F_{1}\left(Q^{2}\right)+F_{2}\left(Q^{2}\right) .
\end{aligned}
$$


In this parameterization, $G_{E}$ and $G_{M}$ evaluated at $Q^{2}=0$ are the electric charge and magnetic moments of the particle. One may also extract the electric charge radius via the relation:

$$
\left\langle r_{E}^{2}\right\rangle=-\left.6 \frac{d G_{E}\left(Q^{2}\right)}{d Q^{2}}\right|_{Q^{2}=0} .
$$

The magnetic moments and electric charge radius are well known low energy observables, and thus help to quantify the low $Q^{2}$ predictions for the electromagnetic form factors in the NJL Model, where it is expected pions will contribute most.

Pion loop corrections were also implemented by Cloët et. al. in their previous work [2], but the method by which those corrections were implemented differs from what is done in this work.

\section{The Light Front Cloudy Bag Model}

The Light Front Cloudy Bag Model (LFCBM) is a quark model which incorporates the effects of the pion cloud [4,5] and was first developed by Miller in 2002. Miller's model is based on an earlier quark model by Schlumpf [6], to which Miller calculated pion corrections using a pseudoscalar pion-nucleon effective hadronic interaction of the form

$$
\mathscr{L}_{\text {int }}=-i g_{N N \pi} \bar{\psi}_{N} \gamma_{5} \vec{\tau} \cdot \vec{\pi} \psi_{N}
$$

where $\vec{\pi}=\left(\pi^{+}, \pi^{-}, \pi^{0}\right)$, and $\pi^{ \pm}=\left(\pi_{1} \mp i \pi_{2}\right) / \sqrt{2}$. Since the bare quark model calculation and the pionic corrections are calculated separately, it is possible to use the chiral correction equations in this work. After minimal substitution, one obtains three Feynman diagrams at first loop order:

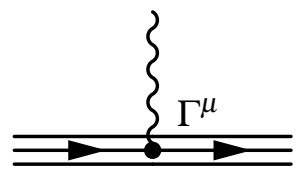

(a)

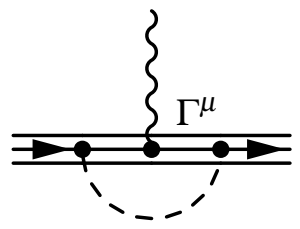

(b)

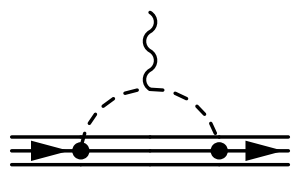

(c)

Figure 1: Feynman diagrams which contribute to the calculation of electromagnetic form factors.

The first diagram is simply the quark model result, while the chiral corrections to these form factors are provided by diagrams shown in Figures $1 \mathrm{~b}$ and $1 \mathrm{c}$. As the name of the model suggests, these equations are evaluated on the light front, but importantly, since the form factors $F_{1}$ and $F_{2}$ are Lorentz invariant scalar functions, it is entirely consistent to take the results of the NJL model as inputs here. The results of that work are summarized here as ${ }^{1}[5]$ :

$$
F_{i}^{N}\left(Q^{2}\right)=Z\left[F_{i, a}^{N}\left(Q^{2}\right)+F_{i, b}^{N}\left(Q^{2}\right)+F_{i, c}^{N}\left(Q^{2}\right)\right],
$$

where $i=1,2, N=p, n, a, b$ and $c$ refer to diagrams $1 \mathrm{a}, 1 \mathrm{~b}$ and $1 \mathrm{c}$, respectively, and $Z$ is the wavefunction renormalisation constant, defined to ensure that the charge of the proton is unity. One may show that this is equivalent to requiring that

$$
F_{1}^{p}(0)=Z\left[F_{1, a}^{p}(0)+F_{1, b}^{p}(0)+F_{1, c}^{p}(0)\right]=1 .
$$

\footnotetext{
${ }^{1} \mathrm{~A}$ version of these equations also exists in [4], but there are several small changes in definitions of equations.
} 
Evaluation of diagrams given in Figures $1 \mathrm{~b}$ and $1 \mathrm{c}$ lead to

$$
\begin{aligned}
F_{1, b}^{N}\left(Q^{2}\right)= & \frac{g_{N N \pi}^{2}}{(4 \pi)} \int_{0}^{1} d x x \int \frac{d^{2} L}{(2 \pi)^{2}}\left[F_{1}\left(Q^{2}\right)\left(L^{2}+x^{2} m_{N}^{2}-\frac{1}{4} x^{2} Q^{2}\right)-F_{2}\left(Q^{2}\right)\left(\frac{x^{2} Q^{2}}{2}\right)\right] \\
& \times \frac{1}{D\left(\vec{L}_{+}^{2}, x\right) D\left(\vec{L}_{-}^{2}, x\right)}, \\
F_{2, b}^{N}\left(Q^{2}\right)= & \left.-\frac{g_{N N \pi}^{2}}{(4 \pi)} \int_{0}^{1} d x x \int \frac{d^{2} L}{(2 \pi)^{2}}\left[F_{1}\left(Q^{2}\right)\left(2 x^{2} m_{N}^{2}\right)+F_{2}\left(Q^{2}\right)\left(L^{2}+x^{2} m_{N}^{2}-\frac{1}{4} x^{2} Q^{2}\right]\right)\right] \\
& \times \frac{1}{D\left(\vec{L}_{+}^{2}, x\right) D\left(\vec{L}_{-}^{2}, x\right)},
\end{aligned}
$$

where $F_{1}$ and $F_{2}$ are given as

$$
F_{i}=\left\{\begin{array}{l}
2 F_{i, a}^{n}+F_{i, a}^{p}, \text { for the proton } \\
2 F_{i, a}^{p}+F_{i, a}^{n}, \text { for the neutron }
\end{array},\right.
$$

and $g_{N N \pi}$ is the nucleon-pion coupling constant. In this work, it is taken that $Z g_{N N \pi}^{2} /(4 \pi)=13.5$. $D$ is given as

$$
D\left(l_{\perp}, x\right)=l_{\perp}^{2}+x^{2} m_{N}^{2}+(1-x) m_{\pi}^{2},
$$

where $\vec{L}_{ \pm}=\vec{L}_{\perp} \pm \frac{1}{2} x \vec{q}_{\perp}$. Evaluating the diagram in Figure 1c leads to

$$
\begin{aligned}
& F_{1, c}^{N}\left(Q^{2}\right)=\frac{g_{N N \pi}^{2}}{(4 \pi)} I_{\tau} F_{\pi}\left(Q^{2}\right) \int_{0}^{1} d x x \int \frac{d^{2} K}{(2 \pi)^{2}}\left[K^{2}+x^{2} m_{N}^{2}-\frac{1}{4}(1-x)^{2} Q^{2}\right] \times \frac{1}{D\left(\vec{L}_{+}^{2}, x\right) D\left(\vec{L}_{-}^{2}, x\right)}, \\
& F_{2, c}^{N}\left(Q^{2}\right)=\frac{g_{N N \pi}^{2}}{(4 \pi)} I_{\tau}\left(2 m_{N}^{2}\right) F_{\pi}\left(Q^{2}\right) \int_{0}^{1} d x x^{2}(1-x) \int \frac{d^{2} K}{(2 \pi)^{2}} \times \frac{1}{D_{N}\left(\vec{L}_{+}^{2}, x\right) D_{N}\left(\vec{L}_{-}^{2}, x\right)},
\end{aligned}
$$

where the nucleon-pion isospin coupling $I_{\tau}$ is given as

$$
I_{\tau}=\left\{\begin{array}{l}
2, \text { for the proton } \\
-2, \text { for the neutron }
\end{array},\right.
$$

and $\vec{K}_{ \pm}=\vec{K}_{\perp} \pm \frac{1}{2}(1-x) \vec{q}_{\perp}$. Note that these equations as stated above are divergent, and require a regularisation prescription to render them finite. In this work, two regularisation prescriptions are considered. They are the Pauli-Villars and $t$-Dependent Form Factor regularisation prescriptions, respectively introduced to the formally divergent integral $I$ as

$$
I_{\mathrm{FF}}=\int \frac{d^{4} k}{(2 \pi)^{4}} f\left(k, m_{N}, m_{\pi}\right)|F(k)|^{2},
$$

and

$$
I_{\mathrm{PV}}=\int \frac{d^{4} k}{(2 \pi)^{4}}\left[f\left(k, m_{N}, m_{\pi}\right)-f\left(k, m_{N}, \Lambda\right)\right],
$$

where the $t$-dependent form factor $F$ is given (in light front coordinates) as:

$$
F\left(\vec{k}_{\perp}, x\right)=\exp \left[-\frac{D\left(\vec{k}_{\perp}^{2}, x\right)}{(1-x) \Lambda^{2}}\right],
$$

and $\Lambda$ parameterises the cutoff scale. 


\subsection{Incorporating the Self Energy}

In calculating the chiral corrections, one is effectively including degrees of freedom previously absent from the system. These degrees of freedom modify bare quantities; indeed this is why the pion cloud is included in the first place. But in a self-consistent calculation, the inclusion of the pion cloud must also lead to corrections to other observables. In particular, the pion cloud must contribute to the nucleon self energy, which is related to the bare nucleon mass $m^{(0)}$ via the wellknown renormalisation condition $m_{N}=m_{N}^{(0)}+\left.\Sigma(\not p)\right|_{\not p=m_{N}}$, where $m_{N}$ and $m_{N}^{(0)}$ are the physical and bare masses of the nucleon, and $\Sigma(\not p)$ is the self energy. Rearranging this equation for the bare mass, one has $m_{N}^{(0)}=m_{N}-\left.\Sigma(\not p)\right|_{\not p=m_{N}}$. In other words, nucleon masses in the bare NJL model should no longer be the physical masses, but rather masses shifted from the physical mass in such a way that after the inclusion of the pion degree of freedom, the self energy interaction acts to lower the mass and yield the expected physical nucleon mass.

Using the effective field theory discussed above, the self energy contribution from the pion to the nucleon must be calculated. This calculation is a trivial one in quantum field theory, and the explicit equations are not included here in the interests of brevity. Since the calculated self energy originates from a second order perturbation, and the nucleon is the ground state in the effective field theory, the self energy must be negative. However, there is no lower bound on the nucleon self energy. Guidance must be taken from other sources.

The Light Front Cloudy Bag Model described above is based on an earlier quark model formulated in the early eighties by Miller, Thomas and Théberge [7, 8], called the Cloudy Bag Model. This model achieved an improvement over many of the predicted observables in the MIT Bag Model by incorporating an explicit pion field. In that quark model, it was found that good fits to other observables were found for a nucleon self energy of between $0.1 \mathrm{GeV}$ and $0.3 \mathrm{GeV}$, and so these are the bounds chosen for this work.

\section{Results}

The bare NJL Model was calculated according to the parameters outlined in Table 1 . This set is motivated in part by ensuring the predicted baryon masses correspond to the known experimental values (see Table 2). In this work, parameters were fitted to the nucleon and $\Xi$ masses. Thus these correspond exactly to their experimental values. One may then use the predicted values of the $\Lambda$ and $\Sigma$ baryons to gauge the goodness of the parameter fit. Although the two predicted values differ slightly from their experimental values, the hierarchy of states is correct, that is, $m_{N}<m_{\Lambda}<m_{\Sigma}<m_{\Xi}$.

\begin{tabular}{cccccccc}
\hline \hline$\Lambda_{I R}$ & $\Lambda_{U V}$ & $m_{l}$ & $m_{s}$ & $G_{\pi}$ & $G_{\rho}$ & $G_{s}$ & $G_{a}$ \\
\hline 0.24 & 0.67 & 0.35 & 0.53 & 14.53 & 8.12 & 4.37 & 3.07 \\
\hline
\end{tabular}

Table 1: Chosen NJL model parameters, where all masses and regularisation parameters are given in units of $\mathrm{GeV}$, and the Lagrangian couplings are given in units of $\mathrm{GeV}^{-2}$.

The nucleon self energy was chosen to be $\Sigma_{N}=0.13 \mathrm{GeV}$. This choice leads to a reasonable prediction of the electric charge radius of the nucleon. Unfortunately, the predicted magnetic 

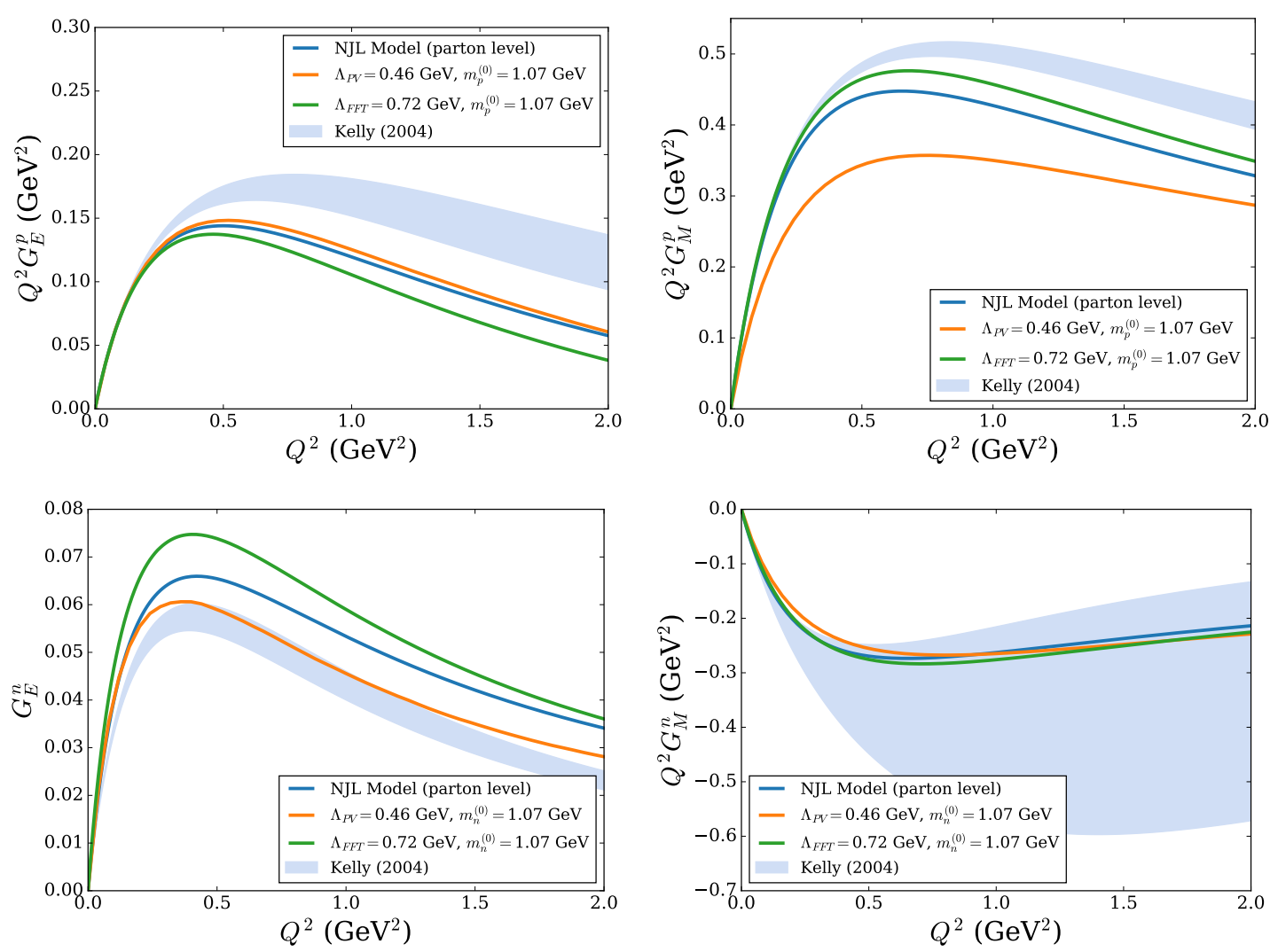

Figure 2: (Colour online) Electromagnetic form factors for the nucleon. Form factors are multiplied by $Q^{2}$ to better show variation between the parameter sets. The shaded region is obtained from uncertainties in the fitted parameters of Kelly's empirical model [9].

\begin{tabular}{ccccc}
\hline \hline & $m_{N}$ & $m_{\Lambda}$ & $m_{\Sigma}$ & $m_{\Xi}$ \\
\hline $\mathrm{NJL}-\Sigma$ & 0.940 & 1.165 & 1.216 & 1.318 \\
Experiment & 0.940 & 1.116 & 1.193 & 1.318 \\
\hline
\end{tabular}

Table 2: Predicted baryon octet masses (after subtracting the hadron's self energy), compared with experimental values. Masses are again given in $\mathrm{GeV}$.

moments are less good, with the best fit found using the $t$-dependent form factor regularisation prescription (coloured orange in plots). Note that both the method employed in this procedings, and the method employed previously [2] are in approximate agreement with the predicted electromagnetic form factors from the empirical parameterization by Kelly [9]. From this it is possible to see that the nucleon system is fairly insensitive to the method by which chiral corrections are implemented.

\section{Conclusion}

This procedings has presented an implementation of chiral corrections to the NJL using the Light Front Cloudy Bag Model. In incorporating an explicit pion field, the nucleon masses deter- 


\begin{tabular}{c|cc|cc}
\hline \hline & \multicolumn{2}{|c|}{$\left\langle r^{2}\right\rangle^{\frac{1}{2}}$} & \multicolumn{2}{c}{$\mu$} \\
& $p$ & $n$ & $p$ & $n$ \\
\hline $\mathrm{NJL}$ & 0.874 & 0.375 & 2.776 & -1.813 \\
$\mathrm{NJL}+\pi(\mathrm{PV}), \Sigma_{N}=0.13$ & 0.867 & 0.381 & 2.047 & -1.554 \\
$\mathrm{NJL}+\pi(\mathrm{FF}), \Sigma_{N}=0.13$ & 0.890 & 0.414 & 2.783 & -1.713 \\
\hline $\mathrm{Exp}$. & 0.875 & 0.335 & 2.793 & -1.913 \\
\hline
\end{tabular}

Table 3: Comparison of the predicted low energy observables (electric charge radius and magnetic moment) to experimental results for the proton and neutron. Experimental results are taken from $[9,10]$. Charge radii are in units of femtometres and magnetic moments are in units of nuclear magnetons $\mu_{N}=e / 2 m_{N}$.

mined in the NJL calculation must be shifted away from their physical masses, by an amount equal to the pion contribution to the self energy. By choosing a new parameter set, it is possible to find a reasonable fit to the low energy observables $\left\langle r_{E}^{2}\right\rangle$ and $\mu$, and also approximately fit an empirical parameterization by Kelly.

\section{References}

[1] M. E. Carrillo-Serrano, I. C. Cloët and A. W. Thomas, SU(3)-flavor breaking in octet baryon masses and axial couplings, Physical Review C 90 (Dec, 2014).

[2] I. C. Cloët, W. Bentz and A. W. Thomas, Role of diquark correlations and the pion cloud in nucleon elastic form factors, Physical Review C 90 (Oct, 2014) .

[3] M. E. Carrillo-Serrano, W. Bentz, I. C. Cloët and A. W. Thomas, Baryon octet electromagnetic form factors in a confining NJL model, Physics Letters B 759 (Aug, 2016) 178-183.

[4] G. A. Miller, Light front cloudy bag model: Nucleon electromagnetic form factors, Physical Review C 66 (Sep, 2002) .

[5] H. H. Matevosyan, G. A. Miller and A. W. Thomas, Comparison of nucleon form factors from lattice QCD against the light front cloudy bag model and extrapolation to the physical mass regime, Physical Review C 71 (May, 2005).

[6] F. Schlumpf, Relativistic constituent quark model for baryons, Doctoral Thesis, .

[7] S. Théberge, A. W. Thomas and G. A. Miller, Pionic corrections to the MIT bag model: The (3, 3) resonance, Physical Review D 22 (Dec, 1980) 2838-2852.

[8] G. Miller, A. Thomas and S. Théberge, Pion-nucleon scattering in the brown-rho bag model, Physics Letters B 91 (Apr, 1980) 192-195.

[9] J. J. Kelly, Simple parametrization of nucleon form factors, Physical Review C 70 (Dec, 2004) .

[10] K. Olive, Review of particle physics, Chinese Physics C 38 (Aug, 2014) 090001. 